\title{
Recombination, cryptic clades and neutral molecular divergence of the microcystin synthetase (mcy) genes of toxic cyanobacterium
} Microcystis aeruginosa

\author{
Yuuhiko Tanabe*1, Tomoharu Sano ${ }^{2}$, Fumie Kasai ${ }^{3}$ and \\ Makoto M Watanabe ${ }^{1}$
}

Address: ${ }^{1}$ Graduate School of Life \& Environmental Sciences, University of Tsukuba, 1-1-1 Tennodai, Tsukuba, Ibaraki 305-8572, Japan, ${ }^{2}$ Laboratory of Intellectual Fundamentals for Environmental Studies, National Institute for Environmental Studies, 16-2 Onogawa, Tsukuba, Ibaraki 305-8506, Japan and ${ }^{3}$ Environmental Biology Division, National Institute for Environmental Studies, 16-2 Onogawa, Tsukuba, Ibaraki 305-8506, Japan

Email: Yuuhiko Tanabe* - y_tanabe@sakura.cc.tsukuba.ac.jp; Tomoharu Sano - sanotomo@nies.go.jp; Fumie Kasai - kasaif@nies.go.jp; Makoto M Watanabe - makoto@sakura.cc.tsukuba.ac.jp

* Corresponding author

Published: 22 May 2009

BMC Evolutionary Biology 2009, 9:1 I5 doi:10.1/86/I47|-2/48-9-II5
Received: 9 July 2008

Accepted: 22 May 2009

This article is available from: http://www.biomedcentral.com//47I-2/48/9/II5

(C) 2009 Tanabe et al; licensee BioMed Central Ltd.

This is an Open Access article distributed under the terms of the Creative Commons Attribution License (http://creativecommons.org/licenses/by/2.0), which permits unrestricted use, distribution, and reproduction in any medium, provided the original work is properly cited.

\begin{abstract}
Background: The water-bloom-forming cyanobacterium Microcystis aeruginosa is a known producer of various kinds of toxic and bioactive chemicals. Of these, hepatotoxic cyclic heptapeptides microcystins have been studied most intensively due to increasing concerns for human health risks and environmental damage. More than 70 variants of microcystins are known, and a single microcystin synthetase (mcy) gene cluster consisting of 10 genes ( $m c y A$ to $m c y J$ ) has been identified to be responsible for the production of all known variants of microcystins. Our previous multilocus sequence typing (MLST) analysis of the seven housekeeping genes indicated that microcystinproducing strains of $M$. aeruginosa are classified into two phylogenetic groups.
\end{abstract}

Results: To investigate whether the mcy genes are genetically structured similarly as in MLST analysis of the housekeeping genes and to identify the evolutionary forces responsible for the genetic divergence of these genes, we used $118 \mathrm{mcy}$-positive isolates to perform phylogenetic and population genetic analyses of mcy genes based on three mcy loci within the mcy gene cluster ( $m c y D, m c y G$, and $m c y)$ ), none of which is involved in the production of different microcystin variants. Both individual phylogenetic analysis and multilocus genealogical analysis of the mcy genes divided our isolates into two clades, consistent with the MLST phylogeny based on seven housekeeping loci. No shared characteristics within each clade are known, and microcystin analyses did not identify any compositional trend specific to each clade. Statistical analyses for recombination indicated that recombination among the mcy genes is much more frequent within clades than between, suggesting that recombination has been an important force maintaining the cryptic divergence of mcy genes. On the other hand, a series of statistical tests provided no strong evidence for selection to explain the deep divergence of the mcy genes. Furthermore, analysis of molecular variance (AMOVA) indicated a low level of geographic structuring in the genetic diversity of mcy.

Conclusion: Our phylogenetic analyses suggest that the mcy genes of $M$. aeruginosa are subdivided into two cryptic clades, consistent with the phylogeny determined by MLST. Population genetic analyses suggest that these two clades have primarily been maintained as a result of homology-dependent recombination and neutral genetic drift. 


\section{Background}

Microcystins are a family of cyclic heptapeptides consisting of seven characteristic amino acids (Fig. 1). Exposure to microcystins poses a severe health risk for both humans and animals, primarily because of hepatotoxicity. Accidental ingestion of water contaminated with microcystins causes acute hepatitis due to the inhibition of protein phosphatase 1 (PP1) and PP2A in hepatocytes, and the possible involvement of microcystins in tumor promotion has also been suggested [1]. Although a number of cyanobacterial genera (e.g., Anabaena, Planktothrix, Nostoc) are known to produce microcystins, the primary producer of microcystins is the water-bloom-forming cyanobacterium Microcystis aeruginosa that is often found in eutrophic freshwater environments such as ponds, lakes, and reservoirs worldwide.

More than 70 structural variants of microcystin with varying levels of toxicity have been reported [2]. Most of these variants differ from each other at the second $(X)$ and/or fourth $(Z)$ amino acid position in the cyclic heptapeptide. Another form of variant in which one or two amino acids are demethylated is also often encountered (Fig. 1). Many strains of $M$. aeruginosa are known to produce more than two variants of microcystins [3]. A single microcystin synthetase ( $m c y$ ) gene cluster (Fig. 1) has been shown to be responsible for all structural variants of microcystins $[4,5]$. The product of the mcy gene cluster is a large multienzyme complex of mixed polyketide synthase (PKS) and nonribosomal peptide synthetase (NRPS) modules.

The mcy gene cluster of $M$. aeruginosa comprises 10 genes, $m c y A$ to $m c y J$, nine of which encode catalytic domains for microcystin synthesis $[4,5]$. By contrast, the product of $m c y H$ is hypothesized to be involved in intra- (or extra-) cellular transportation of microcystins [6]. As in other NRPSs, the products of the mcy gene cluster possess the same number of basic "modules" as the number of amino acids incorporated into the non-ribosomal peptide, synthesizing microcystins by a thiotemplate mechanism [7]. Each NRPS module contains an adenylation domain (A domain), a domain for specific amino acid activation, a condensation domain (C domain), a domain for specific amino acid recognition and peptide bond elongation, and

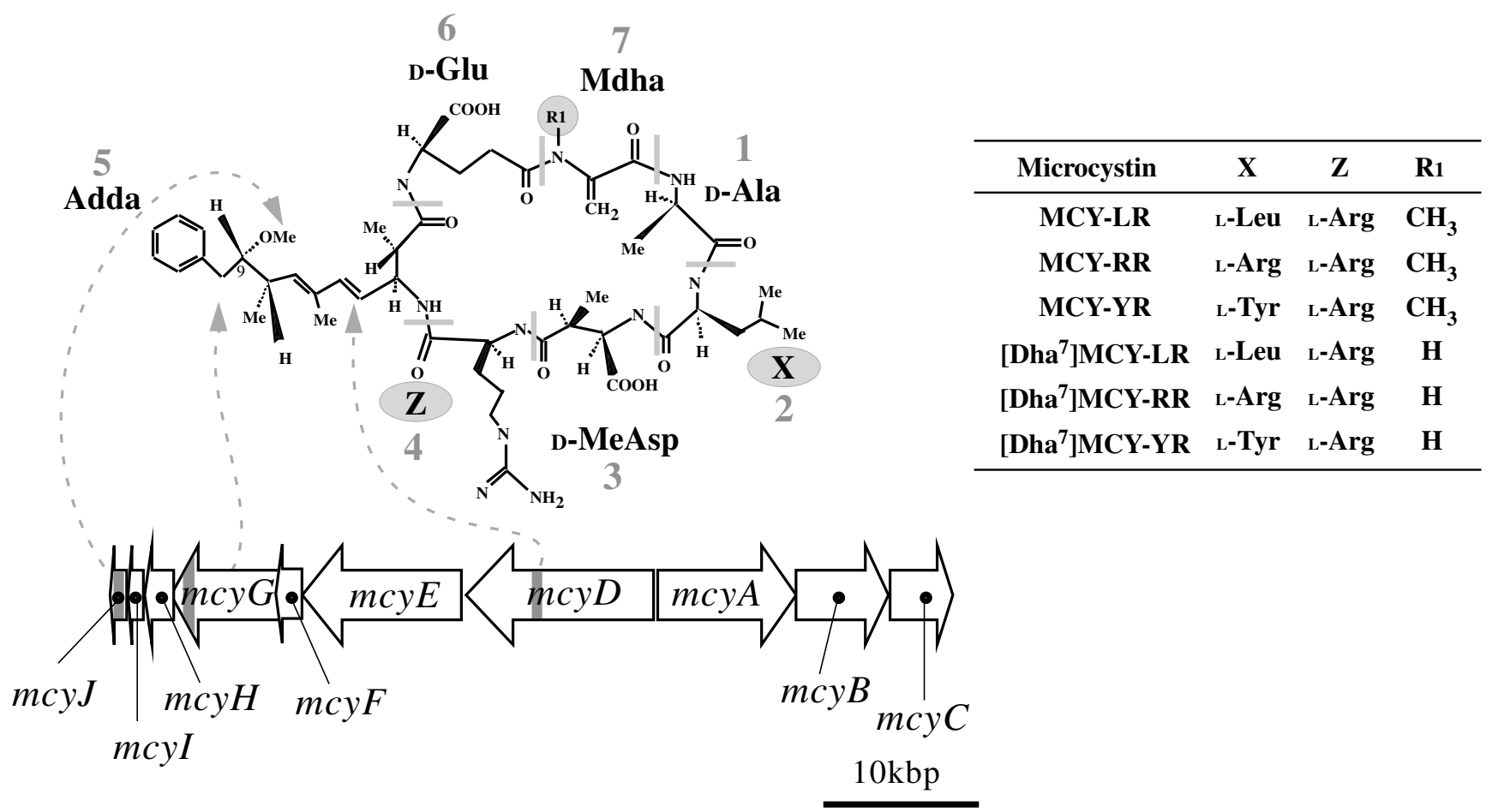

Figure I

Structure of the microcystin synthetase (mcy) gene and microcystins. Structural representation of microcystin variants and the microcystin synthetase ( $m c y)$ gene cluster [5]. General numbering of amino acids is indicated in gray. Arrows indicate the proposed involvement of the product of each mcy marker locus in the incorporation and/or modification of each amino acid into the microcystin. Note that the amino acids $(X$ and $Z)$ and groups $\left(R_{I}\right)$ highlighted in gray are variable. Microcystin is abbreviated as "MCY" in the right-hand table. Abbreviations for three uncommon amino acids in microcystins are as follows: $D^{-M e A s p, ~} D^{-e r y t h r o-~} \beta$-methylaspartic acid; Adda, (2S, 3S, 8S, 9S)-3-amino-9-methoxy-2,6,8-trimethyl- I0-phenyldeca-4,6dienoic acid; MdhA, N-methyldehydroalanine. The positions of PCR primers in the mcy genes are indicated by a gray box. 
a peptidyl carrier protein (PCP). Similarly, the PKS-coding components of the mcy gene cluster contain genes encoding type I PKS modules consisting of a $\beta$-ketoacyl synthase (KS domain), an acyltransferase (AT domain), $\beta$-ketoacyl reductase (KR domain), a dehydratase (DH domain), and an acyl carrier protein (ACP). Other optional domains for tailoring enzymes are also present in the mcy gene cluster.

Given that only a single mcy gene cluster is present in the genome of strains producing two or more variants of microcystins $[4,5,8]$, it has been suggested that the structural variation of microcystins differing in amino acid composition is due to nonspecific amino acid recognition by A domains encoded by the mcy genes rather than differences in the primary structures of $m c y$ [3]. Because the two NRPS modules encoded by the first part of $m c y B(m c y B 1)$ and $m c y C$ are responsible for the variable amino acids in microcystins ( $\mathrm{X}$ and $\mathrm{Z}$, respectively), most previous work investigating the genetic diversity of $m c y$ has focused on these two genes [3,9-11]. Interestingly, it has been demonstrated that gene conversion between A domains, but not $\mathrm{C}$ domains, encoded by $m c y B 1$ and $m c y C$, can explain the difference in the production of specific microcystin variants in a number of strains $[9,10]$. Positive selection at the codon near the binding pocket of the A domain might have an impact on the genetic diversity of $m c y C$ [11]. These results suggest that variation of microcystin composition might be genetically structured to some extent. An important finding has been the recombinational replacement of A domains encoded by $m c \gamma B 1$ with domains from phylogenetically distant origins, probably occurring as a result of horizontal transmission [10]. This finding questions the validity of using A domains, or other redundant domains of PKS and NRPS, to investigate the overall phylogenetic relationships of the $m c y$ gene cluster. Moreover, it has also been demonstrated that both intra- and intergenic recombination significantly contributes to the overall genetic diversity of the mcy genes [12]. Recent occurrences of recombination can significantly bias the result of phylogenetic analysis because they decouple genetic similarity from evolutionary history. Therefore, multiple and conservative regions of the $m c y$ locus should be used as genetic markers to understand better the evolutionary relationships and genetic diversity of the $m c y$ genes as a whole.

Previously we demonstrated that toxic strains are divided into two lineages, group A and group B, based on multilocus phylogenetic analysis of the seven housekeeping genes (MLST) [13]. This finding raises the following two questions: 1) Are mcy genes structured similarly to the housekeeping genes? Or did they evolve independently from the other genes in the genome of M. aeruginosa? 2) Which evolutionary forces are responsible for the observed genetic divergence of the mcy genes? (e.g., are the mcy genes structured as a result of selective sweep or neutral genetic drift following geographic isolation?) To address these issues, we have analyzed the mcy genes from 118 toxic and nontoxic strains of M. aeruginosa.

We performed a suite of phylogenetic and population genetic analyses of the mcy genes using three "conservative" loci within the mcy gene cluster as markers (designated "mcyMLST" by analogy with MLST). MLST is a multilocus sequence-based genotyping protocol that has been widely used to investigate the population structures of a broad spectrum of microbial species ranging from bacteria and archaea to eukaryotic microbes $[14,15]$. A conventional MLST analysis based on the seven housekeeping loci [13] was also performed to compare it with the results of the mcyMLST analysis. We measured the microcystin composition of each strain to investigate the possible correlation between mcy genetic divergence and microcystin composition. Our results show how evolutionary forces, particularly recombination and genetic drift, have contributed to the genetic divergence of $m c y$ genes.

\section{Methods}

\section{Strains, culture, isolation and DNA extraction}

The 196 strains of $M$. aeruginosa used in this study are listed in Additional file 1. They include 11 toxic strains that we previously characterized using $m c y A, m c y D, m c \gamma G$, and $m c y J$ [12], $75 m c y G$-positive and $78 m c y G$-negative strains used in our previous report [13], and 32 toxic strains recently isolated in 2004-2006. The novel 32 strains were isolated by the following protocol. Colonies of M. aeruginosa were picked from field water samples by using a glass micropipette under a microscope, and cultured in a 1:1 mixture of liquid MA medium [16] and autoclaved field water filtrated using a nylon net filter with $0.22 \mu \mathrm{m}$ pore size (Millipore). From these "crude" cultures, a single cell was picked to establish a clonal strain by the same micromanipulation procedure used in crude isolation except that MA medium was used for clonal cultures. Of the 196 strains, 164 are currently available at MCC-NIES (Tsukuba, Japan) and the 32 newly isolated strains will be available at MCC-NIES in the future. Cultures were grown in $10 \mathrm{ml}$ of MA medium at $25^{\circ} \mathrm{C}$ for 1-3 weeks under a 12:12 L/D cycle with a photon density of $15 \mu \mathrm{mol} \mathrm{m} \mathrm{m}^{-2} \mathrm{~s}^{-1}$. Genomic DNA was extracted and purified by a FastDNA ${ }^{\oplus}$ kit (Q-BIOgene).

\section{Multilocus sequencing typing (MLST) of seven housekeeping genes}

The published MLST protocol for M. aeruginosa [13] was employed to genetically characterize the newly isolated strains. Amplified PCR fragments were purified by EXOSAP-IT (USB) and were sequenced in both directions by a DTCS Quick start Kit and a CEQ8000 autosequencer (Beckman Coulter). Following the standard procedure of MLST, each different allele for each locus is assigned a dif- 
ferent arbitrary number, and the unique combination of seven allele numbers ("allelic profile") unambiguously defines a strain's sequence type ("ST"). The MLST sequence data of the 164 strains that we previously characterized [13] are available at the DDBJ database [DDBJ:AB324850-325402]. Newly determined MLST sequence data have been deposited in the DDBJ database [DDBJ: $\underline{\text { AB465739- }}$ 465899].

\section{mcy multilocus sequencing typing (mcyMLST)}

Three loci, $m c y D, m c y G$, and $m c y J$, encoding parts of the DH domain (involved in trans double-bond formation during the polyketide chain elongation of Adda), the A domain (involved in activation of phenylacetate, the precursor of Adda), and $\boldsymbol{o}$-methyltransferase (involved in $\boldsymbol{o}$ methylation of the $\mathrm{C}_{9}$ of Adda), respectively, were selected for the multilocus $m c y$ gene sequence typing ("mcyMLST"). Each locus is expected to be ca. 550 bps in length. Note that these three loci were selected on the basis of three criteria: 1) the domains encoded by these loci are not directly involved in the structural variation of microcystins (at least those identified in this study); 2) the sequences of these loci have diverged sufficiently from other regions of the mcy gene cluster to exclude the possible misamplification of similar but different domains by PCR; 3 ) there is no evidence that these loci have been replaced with the genes of phylogenetically distant organisms through horizontal transmission (as encountered in the A domain of $m c y B 1$ [10]). Each locus was PCR-amplified using published primers (DF/DR, GF/GR, and JF/JR) and optimal reaction conditions [12]. Purification and sequencing reactions of the amplified PCR fragments were performed according to the protocol described above for MLST. As in MLST, each different allele for each mcyMLST locus was assigned a different arbitrary number, thereby creating an "allelic profile" that unambiguously defined a strain's "mcyST" (Additional file 1). Sequence data of 11 strains that we previously characterized are available at the DDBJ database [DDBJ:AB110114-110146]. Newly determined sequence data have been deposited in the DDBJ database [DDBJ: $\underline{\mathrm{AB} 444730}$ - $\underline{44852}$ ].

\section{Phylogenetic analysis}

For phylogenetic analysis, the most appropriate models of the DNA sequence evolution of each mcyMLST locus were selected by a hierarchical likelihood ratio test (hLRT) using MODELTEST version 3.7 [17]. Using PAUP* version 4.0b10 [18], neighbor-joining (NJ) phylogenetic trees were constructed on the basis of the maximum-likelihood (ML) distance calculated from the inferred model and parameters. NJ bootstrap (NJBP) analyses were also performed to assess the statistical confidence of nodes on the basis of alignments generated by 1,000 resamplings of the data with the same DNA substitution models used for phylogenetic reconstruction. Bayesian ML phylogenetic reconstruction was performed with Mr. Bayes version
3.1.2 [19]. Using the DNA evolution model chosen by MODELTEST and the $\mathrm{NJ}$ tree as a starting tree, two independent runs were performed, each with four chains for $5,000,000-7,500,000$ generations (where the convergence diagnostics for each gene hit a stop value of 0.01 ), in which trees were sampled every 100 generations. Statistical confidence for branch support was assessed by posterior probability (PP) estimated from the 50\% majority consensus tree after discarding the burn-in phase of $1,250,000-1,875,000$ generations (corresponding to onefourth of the generation of each run). Phylogenetic analysis of the seven housekeeping genes was performed in the same way as that for $m c y$ genes, except that the seven genes were concatenated prior to analysis, and each run was performed for 3,000,000 generations in Bayesian phylogenetic inference (due to computational limitation).

Using ClonalFrame version 1.1 [20], multilocus genealogies and a suite of population genetic parameters to account for the given $m c y$ data were inferred. The 50\% majority consensus genealogy was generated from the posterior samples of the last 50,000 generations at a thinning interval of 100 after discarding the burn-in phase of first 50,000 generations.

\section{Population genetic analysis}

Estimates of the parameters for DNA sequence divergence, gene diversity $h=[n /(n-1)]\left(1-\Sigma p_{i}{ }^{2}\right)$ (where $n$ is the number of samples, and $p_{i}$ is the relative frequency of ith allele), nucleotide diversity $\pi[21]$, and a test for neutrality by Tajima's $D[22]$ were performed with DnaSP version 4.00 [23]. Coalescent-based estimates of the population recombination rate $(\rho=2 \mathrm{Ner}$, where $\mathrm{Ne}$ is the effective population size, and $r$ is the recombination rate per locus per generation) and population mutation rate $(\theta=2 \mathrm{Ne} \mu$, where $\mu$ is the mutation rate per locus per generation), minimum numbers of recombination $\left(R_{M^{\prime}}[24]\right)$, and statistical probabilities of likelihood permutation test (LPT, [25]) were calculated by LDhat version 2.1 [25]. Multilocus linkage disequilibrium was assessed by the standardized index of association $\left(I_{\mathrm{A}} \mathrm{s},[26]\right)$ using the program START version 2 [27]. $I_{\mathrm{A}} \mathrm{S}$ is a standardized measure of $I_{\mathrm{A}}$ [28], ranging from 0 (panmixia) to 1 (absolute linkage disequilibrium). The statistical significance of non-zero values of $I_{\mathrm{A}} \mathrm{S}$ was inferred on the basis of a comparison of those values estimated from 1,000 randomized datasets under a null hypothesis of panmixia. The phi $(\Phi)$-test (a robust statistical test for recombination [29]) was performed with Splitstree version 4.8 [30]. Maximum likelihood-based tests for phylogenetic congruence between loci [31] were performed with PAUP*. Analysis of molecular variance (AMOVA) was performed with ARLEQUIN version 3.1 [32]. The McDonald-Kreitman test [33] was performed with DNASP. PAML version 3.14 [34] was used to search for sites under positive selection. Possible positively selected sites were also investigated on the basis of 
516 physicochemical criteria with TreeSAAP version 3.2 [35], following the standard protocol except that the statistical significance level was set to $0.001(P<0.001)$.

\section{Microcystin analysis}

To a 10-ml culture of each $M$. aeruginosa strain, $0.5 \mathrm{ml}$ of acetic acid was added and the mixture was ultrasonicated for 15 minutes. After centrifugation (3,000 rpm, $15 \mathrm{~min})$, the supernatant was collected and the remnant pellet was further extracted with $1.5 \mathrm{ml}$ of methyl alcohol by the same procedure as the initial extraction. To the combined supernatant, distilled water was added to $20 \mathrm{ml}$. The diluted extract was passed through a conditioned ( $1 \mathrm{ml}$ of $100 \%$ methyl alcohol and $1 \mathrm{ml}$ of distilled water) Inertsep RP-1 cartridge (GL Science). The cartridge was washed with $20 \%$ methanol aqueous solution, and then eluted with $0.5 \mathrm{ml}$ of $80 \%$ methanol aqueous solution. The eluate was diluted with $0.5 \mathrm{ml}$ of distilled water, and applied to high-performance liquid chromatography (HPLC) using an LC-10A system (Shimadzu; column: Agilent Eclipse XDB RP-18, $2.1 \times 150 \mathrm{~mm}$; solvent: $60 \%$ methanol in $50 \mathrm{mM}$ phosphate buffer, $\mathrm{pH} 3.0$; flow rate: $0.2 \mathrm{ml} /$ min; detection: photodiode array detector). Microcystin variants were identified in comparison with authentic samples in the case of microcystins -LR, -YR, and -RR. Other microcystin variants were identified by liquid chromatography electrospray ionization mass spectrometry (LC ESI-MS) using an LCMS-2010A system (Shimadzu).

\section{Results}

\section{Phylogenetic analysis of the seven housekeeping genes}

The results of the phylogenetic analysis of the seven housekeeping (MLST) loci is shown in Fig. 2. Overall, the results were consistent with those of our previous study [13]. The most toxic strains fell into two lineages, termed group A and group B. The statistical support for group A was weak, whereas that for group $B$ was moderate to strong. In group A, all but one strain represented by ST55 were toxic (microcystin-producer), whereas toxic and non-toxic strains coexisted in group B. Three toxic strains represented by ST23, ST57 and ST95 belonged to neither group A nor group $\mathrm{B}$, forming a distinct monophyletic lineage (designated group "X"). The location of ST40 was ambiguous and varied according to the phylogenetic method used, a feature that was also encountered in our previous study [13]. Three distinct non-toxic clades (groups C, D, and E) were again recovered in this phylogenetic analysis.

\section{mcy multilocus sequence typing (mcyMLST)}

The results of the mcyMLST analysis are shown in Additional file 1 . All sets of primers successfully recovered the three mcy loci from all toxic and mcyG-positive isolates included in this study. By contrast, the primer sets for $m c y D$ and $m c y J$ failed to amplify these genes from any of the $m c y G$-negative strains that we identified in our previous study [13]. The amplified loci of $m c y D, m c y G$, and $m c y J$ were 550, 449, and 552 bp, respectively, in length. Neither insertions nor deletions were found within the sequences of $m c y G$ and $m c y J$, whereas an insertion of $3 \mathrm{bp}$ was found within one $m c \gamma D$ genotype (allele number 18 of $m c y D$ in $m c y S T 25)$ representing five strains. All sequences could be unambiguously aligned (note that the 3-bp insertion in allele 18 of $m c y D$ is TTT, which might be a slippage mutation of the prior TTT sequence). For the $118 \mathrm{mcy}$-positive isolates, $51 \mathrm{mcy}$ sequence types ( $\mathrm{mcySTs}$ ) were found. The number of alleles of $m c \gamma D, m c \gamma G$, and $m c y J$ was 27,31 , and 29, respectively.

\section{Phylogenetic analysis of mcy}

The results of the phylogenetic analysis of the individual $m c y$ loci is shown in Fig. 3. In all of the three mcy phylogenetic trees, $M$. aeruginosa strains could be separated into two clades, group A and group B, consistent with the same grouping as in the MLST phylogeny (Fig. 2). This dichotomy was most robustly supported in the phylogenetic tree of $m c y G$ (NJ BP100\%, Bayesian PP 100\%), whereas it was moderately supported in the $m c y D$ and $m c y J$ phylogenies. Four mcySTs (mcyST10, mcyST11, mcyST24, and mcyST46) showed discordant placements between the two groups (A and B) depending on the loci used. For example, $m c y$ ST10 was located within group A by $m c y D$ and $m c y G$ analysis, but within group B by $m c y J$ analysis. Such discordance is highly likely to be due to recombination between loci. Therefore we next employed ClonalFrame, a multilocus phylogenetic reconstruction method that takes into account the effect of recombination between and within loci. The results of ClonalFrame, which showed the highest likelihood value of 10 independent MCMC runs, are illustrated in Fig. 4a. Again, two highly supported clades (Bayesian PP > 95\%) were recovered. Four anomalous mcySTs (mcyST10, mcyST11, mcyST24, and mcyST46) identified in the individual $m c y$ phylogenies branched at the midpoint of groups $\mathrm{A}$ and $\mathrm{B}$. Within-group relationships were still poorly resolved, as in the analyses of individual loci.

\section{Genetic diversity and recombination}

Because two distinct groups were identified in the $m c y$ phylogenetic and genealogical analyses, genetic diversity indices were estimated from all of the $m c y$ data and also from subsets of data (groups A and B) (Table 1). The maximum sequence divergence of $m c y D, m c y G$, and $m c y J$ in the whole dataset was 3.6\%, 5.1\%, and $2.9 \%$, that in group A was $3.6 \%, 1.5 \%$, and $2.0 \%$, and that in group B was $0.7 \%, 2.0 \%$, and $2.0 \%$, respectively. For $m c \gamma D$, the genetic diversity differed between groups, being much higher in group A $(S=37, \pi=0.0142)$ than in group B $(S$ $=6, \pi=0.0017)$. For $m c y G$ and $m c \gamma J$, the genetic diversity indices were similar between the groups. 


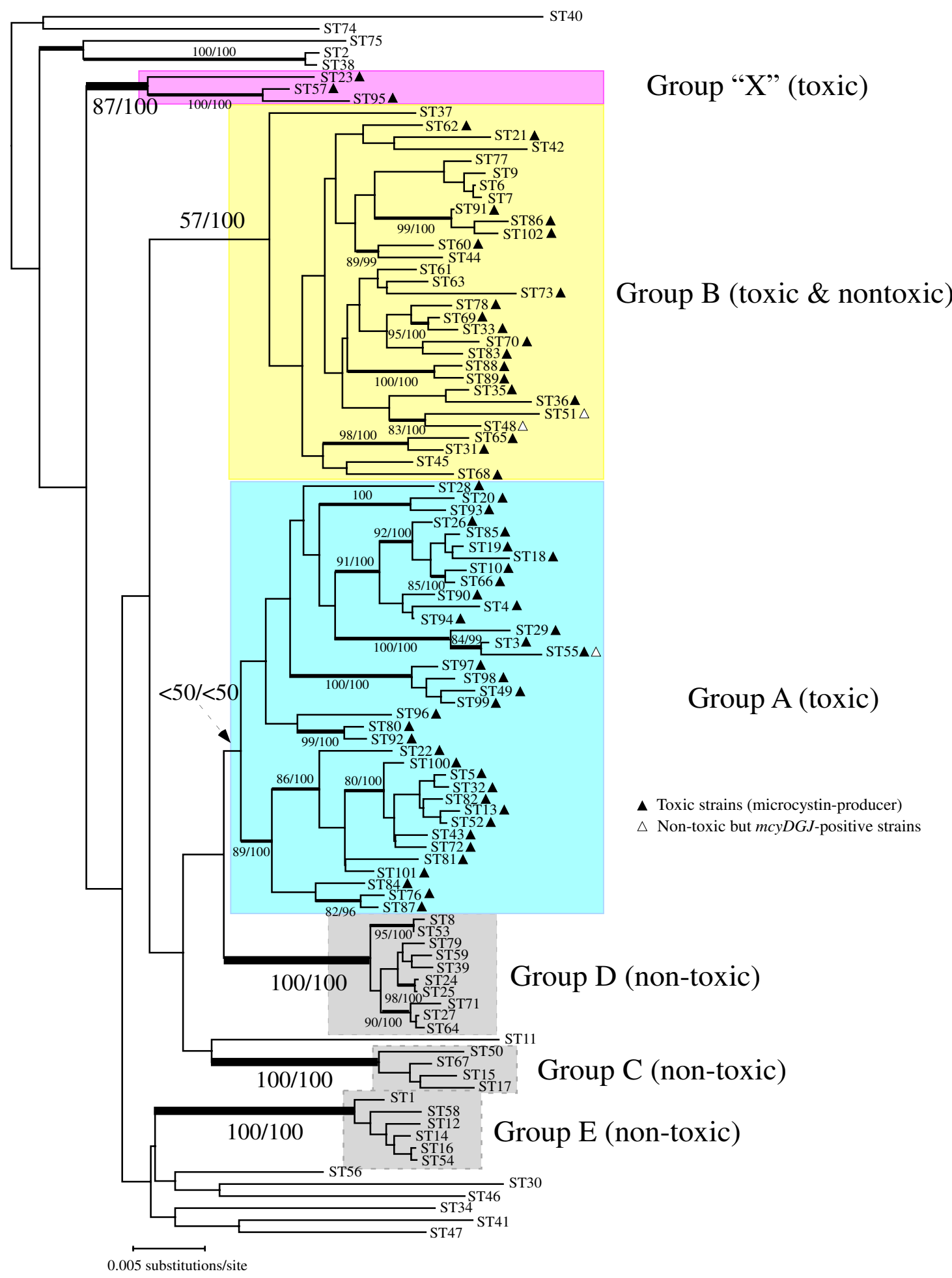

Figure 2

Phylogenetic analysis of the seven housekeeping genes (MLST). Neighbor-joining (NJ) phylogenetic tree of I02 STs of M. aeruginosa based on the concatenated sequences of the seven MLST loci. Branches supported by both NJ bootstrap probabilities (NJBP > 75\%) and Bayesian posterior probabilities (PP > 0.85) are highlighted by thick bars. Statistical values for these branches are indicated (NJ BP, PPxI00). 


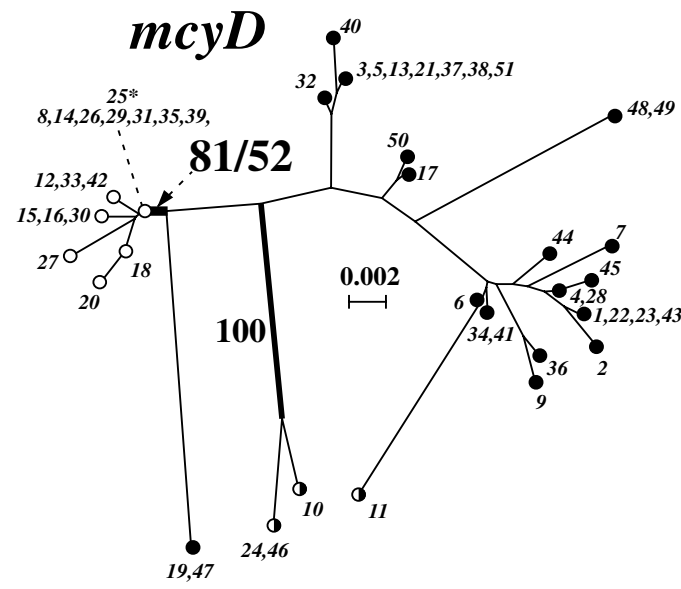

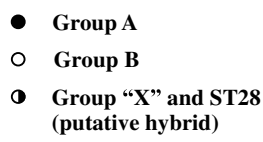
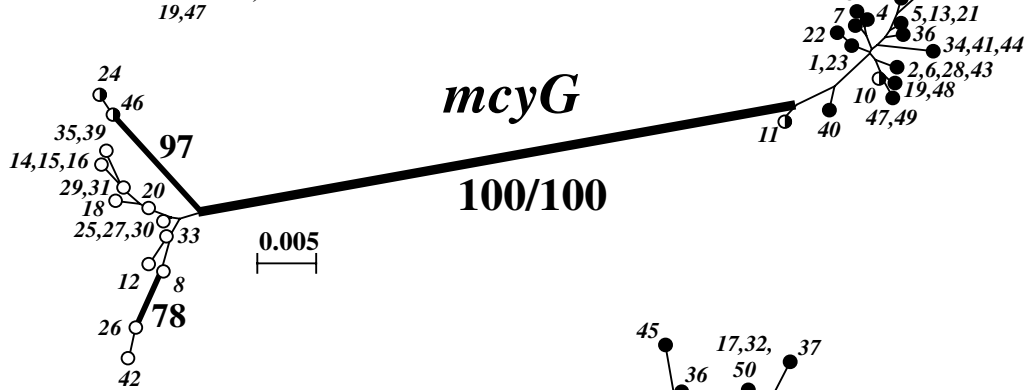

Figure 3

Neighbor-joining tree of $\mathbf{m c y}$ genes. Phylogenetic relationships among the $5 \mathrm{I}$ mcySTs of $M$. aeruginosa based on the $\mathrm{NJ}$ analysis of the individual mcy loci. Statistical values for the branch of the group A-B boundary (in bold) are indicated (NJ bootstrap $[\mathrm{NJBP}] /[$ Bayesian PP] $\times$ 100). In general, within-group relationships were poorly resolved; branches with NJBP > 75\% are highlighted in bold and NJBP values are shown.

Two statistical tests, the likelihood permutation test (LPT) and phi $(\Phi)$ test were performed to explore recombination within loci (Table 1). All tests for intragenic recombination within group A were significant except for one case ( $\Phi$ test of $m c y D$ ), whereas recombination within group B was not significant in any tests except for the LPT of $m c y D$. Consistent with these results, the minimum number of recombination $\left(R_{M}\right)$ was larger in group $\mathrm{A}$ than in group $\mathrm{B}$, and $m c y D$ and $m c y G$ of group B showed no evidence of recombination $\left(R_{M}=0\right)$. Coalescent-based parameter estimates indicated that the population recombination rates $(\rho)$ were generally larger than population mutation rates $(\theta)$. Once again, $\rho$ values for $m c \gamma D$ and $m c y G$ were higher in group A than in group $\mathrm{B}$, but similar between the groups for $m c y J$. On the other hand, statistical tests using the standardized index of association $\left(I_{A}{ }^{S}\right)$ for all samples, group $A$, and group $B$ gave significant positive values $(P<$ 0.01 ) (Table 2). However, $I_{A}$ s using only unique STs gave significant positive values for the whole dataset and group A $(P<0.01)$, whereas the $I_{A} \mathrm{~S}$ of group B was not significantly different from zero $(P=0.167)$. Maximum likelihood-based tests for phylogenetic congruence demonstrated (Table 2$)$ that significant congruence $(P<$ 0.01 ) between the $m c y$ ML trees and randomized trees was present for the whole dataset and group $\mathrm{A}$, but not for group $\mathrm{B}$, indicating that recombination is much more fre- 


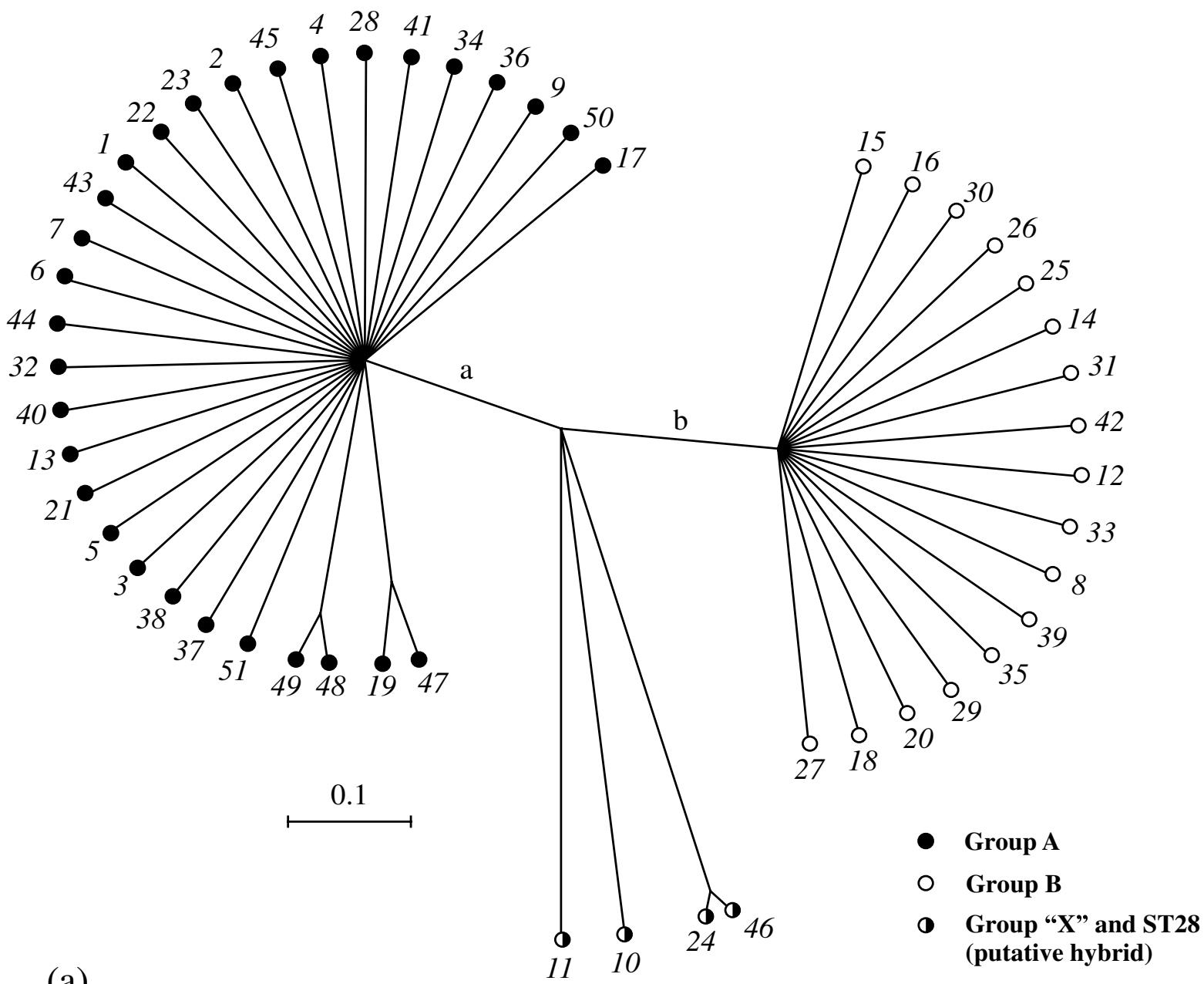

(a)

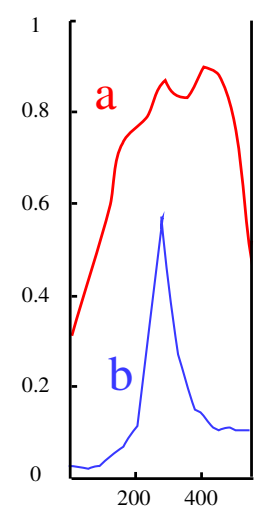

$m c y D$

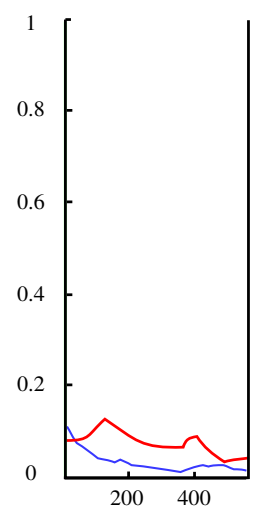

$m c y G$

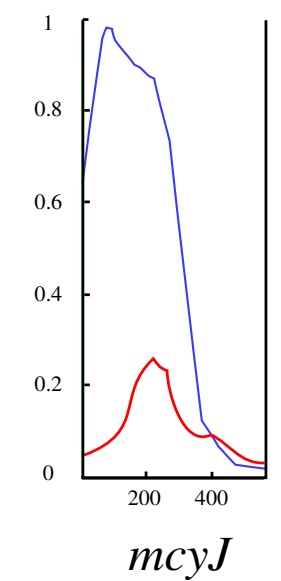

Figure 4

ClonalFrame analysis of mcy genes. (a) A ClonalFrame genealogy of the $5 \mathrm{I}$ mcySTs inferred from the three mcy loci. (b) Genetic representation of the recombination events at the branch of a (red) and $b$ (blue) in the mcy genealogy. X-axis indicates the nucleotide position in the respective mcy loci. Y-axis indicates the posterior probability of recombination. 
Table I: Statistical tests and parameter estimates for mutation and recombination

\begin{tabular}{|c|c|c|c|c|c|c|c|c|c|c|}
\hline & & $S^{\prime}$ & $\pi^{2}$ & $D^{3}$ & $\rho^{4}$ & $\theta^{5}$ & $\rho / \theta$ & $R_{M^{6}}$ & $P_{L P T}{ }^{7}$ & $\boldsymbol{P}_{\phi}{ }^{8}$ \\
\hline \multirow[t]{3}{*}{ mcyD } & All $(n=1 \mid 8)$ & 43 & 0.01529 & 0.133 & 12.6 & 8.047 & 1.56 & 10 & 0.075 & 0.6829 \\
\hline & Group $A^{9}(n=89)$ & 37 & 0.01427 & 0.230 & 14.62 & 7.114 & 2.05 & 10 & $0.037^{*}$ & 0.875 \\
\hline & Group B' $(n=29)$ & 6 & 0.00171 & -1.105 & 4.97 & 1.528 & 3.25 & 0 & $0.020 *$ & 1.00 \\
\hline \multirow[t]{3}{*}{ mcyG } & All $(n=1 \mid 8)$ & 39 & 0.0193 & 1.402 & 1.7 & 6.176 & 0.275 & 5 & 0.298 & 0.5092 \\
\hline & 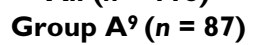 & 14 & 0.00559 & 0.289 & 10.95 & 2.382 & 4.59 & 2 & $0.018^{*}$ & $0.012^{*}$ \\
\hline & Group B' $(n=31)$ & 19 & 0.00635 & -0.921 & 0 & 4.506 & 0 & 0 & 0.08 & 1.00 \\
\hline \multirow[t]{3}{*}{ mcyJ } & All $(n=|| 8)$ & 27 & 0.0128 & 1.174 & 19.5 & 5.053 & 3.85 & 7 & $0.012^{*}$ & $0.0012^{\text {** }}$ \\
\hline & Group $A^{9}(n=84)$ & 16 & 0.00895 & 1.559 & 17.6 & 3.199 & 5.5 & 5 & $0.034 *$ & $0.026^{*}$ \\
\hline & Group B9 $(n=34)$ & 18 & 0.00712 & -0.359 & 18.4 & 4.402 & 4.17 & 4 & 0.362 & 0.428 \\
\hline
\end{tabular}

$* p<0.05$, ** $p<0.01$

I Number of segregating sites.

2 Nucleotide diversity [2I].

3 Tajima's D [22].

${ }^{4}$ Population recombination rate $(=2 \mathrm{Ner})$ estimated from the sites with two alleles.

5 Population mutation rate $(=2 \mathrm{Ne} \mu)$ estimated from the sites with two alleles.

6 Minimum number of recombination based on Hudson and Kaplan [24].

$7 P$-value of likelihood permutation test [25].

${ }^{8} P$-value of $\Phi$ test [29].

${ }^{9} \mathrm{ST}$ s that show discordant group assignment between loci (STI0, II, 24, and 46) are also included.

quent in group B. Overall, the results were consistent but gave different effects of recombination between groups; that is, intralocus recombination is frequent within group A but rare within group $B$, whereas interlocus recombination is frequent within group B but not within group A. Interestingly, the mutation-scaled recombination rate $(\rho /$ $\theta$ ) of $m c y G$ was 20 times larger for group A than for the whole dataset (Table 1). Recombination at a specific branch in the phylogenetic tree can be detected by the program ClonalFrame. For example, our analysis indicated that small segments of $m c y D$ and $m c y J$ are likely to have undergone recombination at the branch of the group A-B boundary (Fig. 4b).

\section{Test for selection}

The inferred parameter values indicative of selection and the results of tests for selection are shown in Table 3. The ratio of nonsynonymous changes per nonsynonymous site $\left(d_{N}\right)$ to synonymous changes per synonymous site
$\left(d_{\mathrm{S}}\right)$ may provide a good measure of adaptive evolution on a given locus because an excess of $d_{\mathrm{N}}$ over $d_{\mathrm{S}}$ (i.e., $d_{\mathrm{N}} / d_{\mathrm{S}}$ $>1$ ) is expected under adaptive evolution. However, the $d_{\mathrm{N}} / d_{\mathrm{S}}$ values of $m c y D, m c \gamma G$, and $m c \gamma J$ were $0.227,0.243$, and 0.169 , respectively, values that are all much smaller than 1 , indicating that purifying selection rather than positive selection is responsible for the genetic diversity of the mcy genes.

Next, we performed several statistical tests to investigate further the presence of selection acting on mcy genes. The Tajima's $D$ statistic indicates selection when it significantly differs from the neutral expectation of $D=0$. As shown in Table 1 , the Tajima's $D$ value for each $m c y$ locus did not significantly differ from zero for the whole dataset or group A or B $(P>0.10)$. To investigate whether selection was responsible for the deep divergence of $m c y$ into groups $\mathrm{A}$ and $\mathrm{B}$, we performed the McDonald-Kreitman $(\mathrm{MK})$ test, which compares the ratio of synonymous to

Table 2: Analyses of interlocus recombination

\begin{tabular}{ccccc}
\hline & & $n^{1}$ & $I_{A}{ }^{S 2}$ & Portion of significant congruence $^{3}$ \\
\hline \multirow{2}{*}{ Total } & All & 118 & $0.656^{*}$ & $6 / 6$ \\
& ST & 51 & $0.188^{*}$ & $0.680^{*}$ \\
Group A & All & 84 & $0.193^{*}$ & $6 / 6$ \\
& ST & 30 & $0.359^{*}$ & $0 / 6$ \\
\hline
\end{tabular}

\footnotetext{
I Number of strains.

2 Standardized index of association [26]. $*, P<0.01$.

3 Maximum likelihood analysis of tree topology congruence based on Feil et al. [3I]. The log-likelihoods of 200 random trees were calculated based on the sequence data of each locus, and compared to those of the ML tree topologies of the other two loci. Log likelihood scores higher than those of 99th percentile of 200 random tree topologies are "significantly congruent" $(P<0.01)$, suggesting the low rate or lack of recombination. Thus, the lower value of the portion of significant congruence indicates the higher rate of recombination.
} 
Table 3: Test for selection

\begin{tabular}{|c|c|c|c|c|c|c|c|}
\hline & $d_{N} / d_{S}{ }^{\prime}$ & $P_{\mathrm{MK}^{2}}$ & $P_{\mathrm{PAML}}{ }^{3}$ & $n_{M}{ }^{4}$ & $n_{M}(A-B)^{5}$ & $n_{T s^{6}}$ & $\mathrm{n}_{\mathrm{TS}}(\mathrm{A}-\mathrm{B})^{7}$ \\
\hline mcyD & 0.2270 & $>0.50$ & $>0.50$ & 0 & 0 & 24 & I \\
\hline mcyG & 0.2437 & $>0.50$ & $<0.001$ & $2(M 2 a)$ & 2 & 15 & 7 \\
\hline mcyJ & 0.1696 & $>0.50$ & $<0.001$ & $2(M 8)$ & 1 & 10 & 1 \\
\hline
\end{tabular}

'Ratio of nonsynonymous substitutions per nonsynonymous sites to synonymous substitutions per synonymous sites based on MO model of PAML. 2P-value of the McDonald-Kreitman test (group A vs. B) [33].

${ }^{3 P}$-values of likelihood ratio tests for both Mla vs. M2a, and M7 vs. M8 in PAML [34]. Significant value indicates the presence of positively selected sites.

${ }^{4}$ Number of codons under positive selection detected by the model showing the highest likelihood (indicated in parentheses) of PAML ( $P<0.05$ ).

${ }^{5}$ Number of codons under positive selection changed on the branch of the group A-B boundary according to PAML using the model described in $\mathrm{n}_{\mathrm{M}}$ column

6 Number of codons under positive selection according to TreeSAAP [35].

${ }^{7}$ Number of codons under positive selection changed on the branch of the group A-B boundary according to TreeSAAP.

non-synonymous polymorphism within groups and that of synonymous to non-synonymous divergence (fixed differences) between groups; these two ratios should be equal under the null hypothesis of neutrality [33]. Again, none of the MK test $P$ values was significant (Table 3 ), and thus did not provide evidence for selection on the branch of the group A-B boundary.

All of the above analyses are based on the average nucleotide polymorphism; however, these kinds of test are not sensitive enough to detect a single or small number of adaptive amino acid changes, which are sometimes responsible for improved fitness of a given gene. Two programs, PAML and TreeSAAP, were therefore used to overcome this problem. The first program PAML uses the same approach as the $d_{\mathrm{N}} / d_{\mathrm{S}}$ consideration but applies it to individual sites on the basis of maximum-likelihood, thus enabling codons under positive selection to be detected. Using PAML, we employed the "site models", and performed two likelihood-ratio tests, a test of M1a (nearly neutral) versus M2a (positive selection), and that of M7 (beta; assuming a beta distribution of $d_{\mathrm{N}} / d_{\mathrm{S}}$ over sites ranging from 0 to 1 ) versus $M 8$ (beta $\& \omega$; the same as $M 7$ with an additional estimate of $\left.\omega=d_{\mathrm{N}} / d_{\mathrm{S}}>1\right)$ to determine any sites under positive selection $\left(d_{\mathrm{N}} / d_{\mathrm{S}}>1\right)$, as suggested by the author [34]. Significant likelihood ratio values were obtained for $m c y G$ and $m c y J(P<0.01)$, but not for $m c y D$ $(P>0.50)$. For each locus $(m c y G$ and $m c y J)$, two codons were identified as positively selected sites by the model that showed the highest log-likelihood (the M2a model for $m c y G$, and M8 model for $m c y J$ ). Although the number was small (up to two), the most likely positively selected sites were substituted on the branch bordering groups A and $\mathrm{B}$.

The second program, TreeSAAP, provides a method to detect an adaptive amino acid change by taking quantitative amino acid properties into account. On the basis of statistical tests for differences in the observed versus the expected change under neutrality in the eight categories of
516 physicochemical criteria (e.g., changes in hydropathy, isoelectric point, polarity, and so on), TreeSAAP identified 24,15 , and 10 sites under positive selection in $m c y D$, $m c y G$, and $m c y J$, respectively; these numbers of sites were much larger than those identified by PAML. TreeSAAP also identified all of the positively selected sites identified by PAML, including the sites at the branch of the A-B boundary. Unlike the result of PAML analyses, the most likely positively selected sites identified in $m c y D$ and $m c y J$ did not change on the branch bordering groups A and $\mathrm{B}$, whereas half of the selected sites in $m c y G$ were identified to have been substituted on the branch of the A-B boundary.

\section{Genetic differentiation}

To investigate the significance of geographic isolation on the genetic diversity of the mcy genes, our strains were partitioned into their geographic origins. Because the inclusion of localities with a small number of strains may bias the result of AMOVA, we included only strains for which more than five isolates were available from a single locality. On the basis of this criterion, nine groups of strains from Lake Barato $(n=6)$, Lake Inba $(n=22)$, Lake Kasumigaura $(n=22)$, Lake Okutama $(n=12)$, Lake Suwa $(n=$ 5), Lake Teganuma $(n=11)$, Ishigaki Dam $(n=5)$ and Kunnma Dam $(n=6)$ were selected and analyzed. The results of AMOVA (Table 4) indicated low but significant genetic structuring among local populations $\left(F_{\mathrm{ST}}=0.212\right.$, $P<0.001)$, although the within-population genetic variance was still high.

\section{Microcystin analysis}

Most strains were found to produce multiple variants of microcystins (Additional file 1). On the basis of the microcystin composition, our strains were divided into eight categories (Fig. 5). Several isolates were found to produce other microcystin variants at a low concentration (less than $10 \%$ of the total amount of microcystins). We did not include them here, because such minor variants are highly likely to have been produced through the occa- 
Table 4: Analysis of molecular variance (AMOVA)

\begin{tabular}{|c|c|c|c|c|c|c|}
\hline Source of variation & d.f. & Sum of squares & Variance component & $\%$ of variation & $F_{\mathrm{ST}}$ & $P$ value \\
\hline Among local populations & 8 & 282.739 & 2.83773 & 21.26 & & \\
\hline Within populations & 73 & 767.395 & 10.51226 & 78.74 & & \\
\hline Total & 81 & 1050.134 & 13.34999 & & 0.212 & $<0.001$ \\
\hline
\end{tabular}

sional misrecognition of amino acids by the substratebinding pocket of the A domain and therefore would appear to be not selectively important.

The most dominant form of microcystin was microcystinLR and -RR, representing 56\% of the total $118 \mathrm{mcy}$-positive isolates. The second most dominant form was microcystin-LR, -RR, and -YR, which accounted for $22 \%$ of the 118 strains. In general, group $\mathrm{B}$ appeared to be more divergent in microcystin composition as compared with group A. For example, demethylated microcystin variants ([Dha $\left.{ }^{7}\right]$ microcystin-LR, -RR, and -YR, Fig. 1) were exclusively present in strains assigned to group B (except for the anomalous mcyST10), although genotypic relationships among the different variants were poorly resolved. Indeed, the difference in microcystin composition between group A and group B was significant $\left(\chi^{2}=44.7\right.$, d.f. $=6, P<0.001)$. Some strains with the same $m c y s T$ were found to produce a different combination of microcystin variants (e.g., mcyST1, see Additional file 1). Once again, it should be noted here that five $m c y$-positive iso-

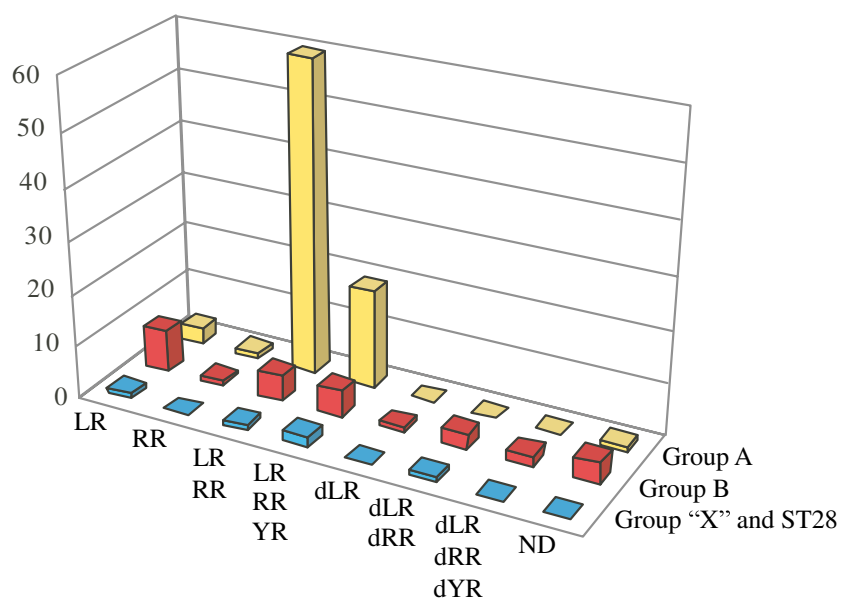

\section{Figure 5}

Microcystin composition. Distribution of microcystin variants in groups $\mathrm{A}, \mathrm{B}$, and " $\mathrm{X}$ " (putative hybrid mcySTs: mcySTI0, mcySTII, mcyST24, and mcyST46). Abbreviations for the microcystin variants are as follows: LR, microcystinLR; RR, microcystin-RR; YR, microcystin-YR; dLR, [Dha ${ }^{7}$ microcystin-LR; dRR, [Dha ${ }^{7}$ microcystin-RR; dYR, $\left[\mathrm{Dha}^{7}\right]$ microcystin-YR; ND, not detected. lates did not produce a detectable level of microcystins [13] (Additional file 1).

\section{Discussion}

Phylogenetic analysis based on the seven housekeeping loci (MLST) indicated that toxic strains are affiliated into two major clades, group A and group B, and an outlier clade group "X" (Fig. 2). This observed subgrouping of toxic strains is largely consistent with our previous MLST phylogeny [13], suggesting that the ability to produce microcystins is genetically structured to some extent. On the other hand, our phylogenetic and multilocus genealogical analyses of the mcyMLST divided our strains into two clades (Fig. 3, 4a). Importantly, these two clades correspond to groups $\mathrm{A}$ and $\mathrm{B}$ in the MLST phylogeny. The congruent phylogenies between the mcy (mcyA, mcyD, $m c y G$, and $m c y E$ ) and housekeeping genes (e.g., $16 \mathrm{~S}$ rRNA) of several microcystin-producing cyanobacterial genera have been demonstrated and suggested to be evidence for the vertical rather than interspecific horizontal transmission of $m c y$ genes $[10,36]$. The overall concordance of group assignment between the mcy and MLST phylogenies in this study suggests that horizontal transmission of mcy between distantly related Microcystis strains is also not so frequent. Interestingly however, strains included in group "X" and ST28 (mcyST11) in group A show discordant phylogenetic placements between groups A and B in the mcy phylogeny depending on the mcy loci used, suggesting that these strains represent the consequence of intergroup horizontal transmissions of $m c y$ genes followed by recombination.

Our population genetic data corroborate our previous finding that recombination is an important force in maintaining the genetic diversity of the mcy genes [12]. Moreover, population recombination rates were larger than mutation rates in general $(\rho / \theta>1$, Table 1$)$, suggesting that recombination is more frequent than point mutation within the $m c y$ genes. Although our data may not be robust owing to the small sample sizes, the relative contribution of recombination and point mutation to the genetic diversity of the mcy genes may be comparable to that of various genes of other highly recombinogenic bacteria, such as Helicobacter pylori and Streptococcus pneumoniae [37]. ClonalFrame assumes that recombined fragments (imported donor sequence) differ from the 
parental segments (original recipient sequence) at a constant percentage of $v$, which can be used to roughly infer the origin of recombined fragments [20]. Our estimate of $v$ of $m c y$ is 0.018 , which is lower than the maximum sequence divergence $(0.050)$ of $m c y$ genes. This result suggests that most recombined segments originated from within $M$. aeruginosa. ClonalFrame also estimated the mean length of recombined segments as $206 \mathrm{bp}$, which is in line with a previous study showing that bacterial recombination can occur within a very short length of DNA $(<1 \mathrm{kbp})$ [38]. The very small size of the imported segments implies that they are subject to various restriction systems [39] discovered within the genome of $M$. aeruginosa [8].

Within clades, recombination was suggested to be frequent, although the effect of recombination appeared to differ between groups and loci. For example, interlocus recombination in group $B$ was so frequent that alleles at different $m c y$ loci were assorted randomly ("panmictic"), whereas the clonal divergence of $m c y$ genes was suggested in group A (Table 2). When we examined intralocus recombination, however, most significant recombination was found for group A, with little recombination for group B (Table 1). The different impact of recombination between two clades might be due to the different forms of genetic exchange and restriction system, because the length of recombined segments is known to be highly dependent on these mechanisms [40].

Importantly, statistical tests indicated that recombination seems to be more substantial within groups than between (Table 1, 2), implying that there is genetic isolation between groups A and B owing to DNA sequence divergence. This result suggests that homology-dependent recombination makes a significant contribution to the strong genetic clustering of $m c y$ genes. In this context, each $m c y$ group may be recognized as a fuzzy analog of "biological species" in higher eukaryotes [41]. Accordingly, strains belonging to group " $\mathrm{X}$ " might represent "hybrids" that could successfully cross over the genetic barrier between the two groups A and B. Bacterial recombination is often mediated by vectors (e.g., phages). Some cyanophages that are infectious to Microcystis have been suggested to have a very narrow host range [42]. Such vector specificity might play a role in augmenting the genetic isolation between the two mcy clades. Finally, the hypothesis that recombination might also play an important role in the diversification of $m c y$ genes should not be dismissed. As shown in Fig. 4b, significant recombination is likely to have occurred on the branch bordering groups $\mathrm{A}$ and $\mathrm{B}$.

Our data suggest that selection has not been an important factor in the genetic divergence of the mcy genes. None of Tajima's D-values was significantly different from the neutral assumption, and the MK test failed to reject the neu- tral divergence of $m c y$ into the two groups A and B. In addition, the PAML and TreeSAAP analyses identified few positively selected sites within the $m c y$ genes on the branch separating groups $\mathrm{A}$ and $\mathrm{B}$. One exception was the TreeSAAP analysis of $m c y G$, which identified seven possible sites under positive selection on the branch of the group A-B boundary. The product of $m c y G$ is probably involved in polyketide chain elongation within the Adda of microcystins [5]. All microcystin variants identified in this study share the same Adda at the corresponding position of the microcystin (Fig. 1), and therefore positive selection at these sites, if present, appears to have little significance with regard to microcystin structure. It is possible that the observed deep divergence of $m c y G$ arose as a result of selection at different but linked mcy genes. If so, it would be expected that these two clades would differ in microcystin composition, which might be thought to confer selective advantages under different (but unknown) ecological conditions. However, analysis of microcystin composition did not support this hypothesis. Although demethylated types of structural microcystin variants are exclusively present in strains in group B (except for $m c y S T 10$ in group " $\mathrm{X}$ "), the most frequently found microcystin compositions (e.g., microcystin-LR and -RR, and microcystin-LR, -RR, and -YR) are present in both groups (Fig. 5), suggesting that mcy genealogy is decoupled from microcystin variation. Similar results have been obtained for the freshwater cyanobacterium Planktothrix, in which discordance between the genetic relationships and compositional trends of nonribosomal peptides is encountered [43]. Moreover, it has been demonstrated that positive selection at the amino acid site neighboring the binding pocket of the A domain of $m c y B$ in Microcystis does not contribute to the production of specific microcystin variants [11]. Unfortunately, the biological role of microcystins has yet to be determined, although several possible functions have been proposed [44-46]. Clarifying the function of microcystins will be critical to evaluate further the importance of selection on the mcy genes.

As described above, strains affiliated to groups A and B in the MLST phylogeny (Fig. 2) are generally clustered in the same groups in the mcy phylogeny (Fig. 3, 4a). We previously suggested that the intraspecies clades found in the MLST phylogeny might represent "ecotypes" [13]. In this context, it is possible that selective sweeps on linked loci outside the mcy genes yielded the structured mcy phylogeny (i.e. a "hitchhiking effect"). For M. aeruginosa, several morphological (e.g., colony morphology [47]) and physiological (e.g., photosynthetic pigments [48]) variations are known. As shown in other cyanobacteria [49], positive selection may favor these characteristics, but none of them is specific to either of the two groups (A and B) in M. aeruginosa (YT, unpublished data). Of course, investigation of previously uncharacterized ecological parameters are needed to rule out the possibility of $m c y$ divergence due to 
a hitchhiking effect. In any event, selection acting on the $m c y$ genes has little impact on their deep divergence with regard to the variation of microcystin composition.

Genetic clustering can arise in the absence of selection where gene flow among populations is restricted due to a geographic barrier. Although a few exceptions are known [50], this possibility has been considered unlikely for microbes including M. aeruginosa for which long-distance dispersal may be easy owing to their small cell size and immense population size [51]. In fact, $M$. aeruginosa strains belonging to different groups in the mcy phylogenies are often isolated from a small amount of water sampled from one location at one time (Additional file 1), suggesting that allopatry is not an important factor in the genetic isolation observed between the two mcy groups. Consistent with this observation, the results of our AMOVA found little geographic contribution to the pattern of genetic variation within the mcy genes (Table 4).

Despite the absence of geographic isolation and selection, we have identified two distinct $m c y$ clusters for which recombination is much more frequent within than between. These results suggest that recombination and neutral genetic drift are primarily responsible for the observed deep divergence of the mcy genes in M. aeruginosa. This pattern of bacterial genetic divergence is in line with recent theoretical results indicating that genotypic clusters can arise and be maintained in the absence of selection or physical isolation when the recombination rate is a negative log-linear function of genetic distance [52,53] and the effective population size $(\mathrm{Ne})$ is extremely large [54]. Although the $\mathrm{Ne}$ of a bacterial population is difficult to appreciate and would be at least much lower than the census population size [53], M. aeruginosa often forms a bloom with extremely large numbers of cells (sometimes exceeding $10^{6} \mathrm{cell} / \mathrm{ml}$ [55]) with a high level of neutral genetic diversity [13], and is thus likely to fulfill the latter condition [54]. Recently, neutral molecular evolution in the natural bacterial population has received more attention than previously, and indeed potential evidence for it has been reported $[56,57]$.

\section{Conclusion}

Our phylogenetic and population genetic analyses of multiple conservative loci within the microcystin synthetase ( $m c y$ ) gene cluster suggested that $m c y$ genes of M. aeruginosa are subdivided into two cryptic clades, which have been primarily generated and maintained as a result of homologydependent recombination and neutral genetic drift.

\section{Abbreviations}

AMOVA: analysis of molecular variance; BP: bootstrap probability; MLST: multilocus sequence typing; NJ: neighbor-joining; PP: posterior probability; ST: sequence type.

\section{Authors' contributions}

YT designed the research, YT and MMW collected samples, YT performed isolation, culturing, DNA experiments, phylogenetic and population genetic analyses, YT and TS performed microcystin analyses, YT, TS, and MMW wrote the paper, and YT, FK, and MMW coordinated the research. All authors read and approved the final manuscript.

\section{Additional material}

\section{Additional File 1}

Strain information. Genotypic and toxin profiles of strains used in this study.

Click here for file

[http://www.biomedcentral.com/content/supplementary/14712148-9-115-S1.pdf]

\section{Acknowledgements}

We thank S. Bounphanmy, National University of Laos, for helping us collect M. aeruginosa samples from Lao P.D.R. This work was supported by the grant of the Institute for Fermentation, Osaka (IFO) to YT.

\section{References}

I. Dittmann E, Wiegand C: Cyanobacterial toxins-occurrence, biosynthesis and impact on human affairs. Mol Nutr Food Res 2006, 50:7-17.

2. Codd GA, Lindsay J, Young FM, Morrison LF, Metcalf JS: Harmful cyanobacteria: from mass mortalities to management measures. In Harmful cyanobacteria Edited by: Huisman J, Matthijs HCP, Visser PM. Dordrecht, The Netherlands: Springer; 2005:I-24.

3. Kurmayer R, Dittmann E, Fastner J, Chorus I: Diversity of microcystin genes within a population of the toxic cyanobacterium Microcystis spp. in Lake Wannsee (Berlin, Germany). Microb Ecol 2002, 43:107-1 I8.

4. Nishizawa T, Asayama M, Fujii K, Harada K, Shirai M: Genetic analysis of the peptide synthetase genes for a cyclic heptapeptide microcystin in Microcystis spp. J Biochem 1999, I 26:520-529.

5. Tillett D, Dittmann E, Erhard M, von Döhren H, Börner T, Neilan BA: Structural organization of microcystin biosynthesis in Microcystis aeruginosa PCC7806: an integrated peptide-polyketide synthetase system. Chem Biol 2000, 7:753-764.

6. Pearson LA, Hisbergues M, Börner T, Dittmann E, Neilan BA: Inactivation of an $A B C$ transporter gene, $m c y H$, results in loss of microcystin production in the cyanobacterium Microcystis aeruginosa PCC 7806. Appl Environ Microbiol 2004, 70:6370-6378.

7. Cane DE, Walsh CT, Khosla C: Harnessing the biosynthetic code: combinations, permutations, and mutations. Science 1998, 282:63-68.

8. Kaneko T, Nakajima N, Okamoto S, Suzuki I, Tanabe Y, Tamaoki M, Nakamura Y, Kasai F, Watanabe A, Kawashima K, et al.: Complete genomic structure of the bloom-forming toxic cyanobacterium Microcystis aeruginosa NIES-843. DNA Res 2007, 14:247-256.

9. Mikalsen B, Boison G, Skulberg OM, Fastner J, Davies W, Gabrielsen TM, Rudi K, Jakobsen KS: Natural variation in the microcystin synthetase operon mcyABC and impact on microcystin production in Microcystis strains. I Bacteriol 2003, 185:2774-2785.

10. Fewer DP, Rouhiainen L, Jokela J, Wahlsten M, Laakso K, Wang H, Sivonen K: Recurrent adenylation domain replacement in the microcystin synthetase gene cluster. BMC Evol Biol 2007, 7: 183 .

II. Tooming-Klunderud A, Fewer DP, Rohrlack T, Jokela J, Rouhiainen L, Sivonen K, Kristensen T, Jakobsen KS: Evidence for positive selection acting on microcystin synthetase adenylation domains in three cyanobacterial genera. BMC Evol Biol 2008, 8:256. 
12. Tanabe Y, Kaya K, Watanabe MM: Evidence for recombination in the microcystin synthetase (mcy) genes of toxic cyanobacteria Microcystis spp. J Mol Evol 2004, 58:633-64I.

13. Tanabe $Y$, Kasai $F$, Watanabe MM: Multilocus sequence typing (MLST) reveals high genetic diversity and clonal population structure of the toxic cyanobacterium Microcystis aeruginosa. Microbiology 2007, 153:3695-3703.

14. Maiden MC, Bygraves JA, Feil E, Morelli G, Russell JE, Urwin R, Zhang $\mathrm{Q}$, Zhou J, Zurth K, Caugant DA, et al:: Multilocus sequence typing: a portable approach to the identification of clones within populations of pathogenic microorganisms. Proc Natl Acad Sci USA 1998, 95:3|40-3|45.

15. Urwin R, Maiden MC: Multi-locus sequence typing: a tool for global epidemiology. Trends Microbiol 2003, I I:479-487.

16. Kasai F, Kawachi M, Erata M, Watanabe MM: NIES-Collection, List of strains, microalgae and protozoa 7th edition. Tsukuba, Japan: National Institute for Environmental Studies; 2004.

17. Posada $D$, Crandall KA: MODELTEST: testing the model of DNA substitution. Bioinformatics 1998, 14:817-8|8.

18. Swofford DL: PAUP* - Phylogenetic analysis using parsimony (*and other methods) version 4. Sunderland, MA: Sianuer Associates; 2002.

19. Ronquist F, Huelsenbeck JP: MRBAYES 3: Bayesian phylogenetic inference under mixed models. Bioinformatics 2003, 19:1572-1574.

20. Didelot $X$, Falush D: Inference of bacterial microevolution using multilocus sequence data. Genetics 2007, 5: I25I-I 266.

21. Nei M: Molecular Evolutionary Genetics New York, NY: Columbia University Press; 1987.

22. Tajima F: Statistical method for testing the neutral mutation hypothesis by DNA polymorphism. Genetics 1989, 1 23:585-595.

23. Rozas J, Sanchez-DelBarrio JC, Messeguer X, Rozas R: DnaSP, DNA polymorphism analyses by the coalescent and other methods. Bioinformatics 2003, 19:2496-2497.

24. Hudson RR, Kaplan NL: Statistical properties of the number of recombination events in the history of a sample of DNA sequences. Genetics 1985, II I:I47-164.

25. McVean G, Awadalla P, Fearnhead P: A coalescent-based method for detecting and estimating recombination from gene sequences. Genetics 2002, 160:1231-1241.

26. Haubold $B$, Hudson RR: LIAN 3.0: detecting linkage disequilibrium in multilocus data. Linkage Analysis. Bioinformatics 2000, 16:847-848.

27. Jolley KA, Feil EJ, Chan MS, Maiden MC: Sequence type analysis and recombinational tests (START). Bioinformatics 200I, I7:|230-|23|

28. Smith JM, Smith NH, O'Rourke M, Spratt BG: How clonal are bacteria? Proc Natl Acad Sci USA 1993, 90:4384-4388.

29. Bruen TC, Philippe $H$, Bryant $D$ : A simple and robust statistical test for detecting the presence of recombination. Genetics 2006, I72:2665-268I.

30. Huson DH, Bryant D: Application of phylogenetic networks in evolutionary studies. Mol Biol Evol 2006, 23:254-267.

3I. Feil EJ, Holmes EC, Bessen DE, Chan MS, Day NP, Enright MC, Goldstein R, Hood DW, Kalia A, Moore CE, et al.: Recombination within natural populations of pathogenic bacteria: shortterm empirical estimates and long-term phylogenetic consequences. Proc Natl Acad Sci USA 2001, 98: 182-187.

32. Excoffier L, Laval G, Schneider S: Arlequin ver. 3.0: An integrated software package for population genetics data analysis. Evolutionary Bioinformatics Online 2005, I:47-50.

33. McDonald $\mathrm{JH}$, Kreitman M: Adaptive protein evolution at the Adh locus in Drosophila. Nature 1991, 35 I:652-654.

34. Yang Z: PAML: a program package for phylogenetic analysis by maximum likelihood. Comput Appl Biosci 1997, 13:555-556.

35. Woolley S, Johnson J, Smith MJ, Crandall KA, McClellan DA: TreeSAAP: selection on amino acid properties using phylogenetic trees. Bioinformatics 2003, 19:67।-672.

36. Rantala A, Fewer DP, Hisbergues M, Rouhiainen L, Vaitomaa J, Börner $T$, Sivonen K: Phylogenetic evidence for the early evolution of microcystin synthesis. Proc Natl Acad Sci USA 2004, I 0 I:568-573.

37. Pérez-Losada M, Browne EB, Madsen A, Wirth T, Viscidi RP, Crandal KA: Population genetics of microbial pathogens estimated from multilocus sequence typing (MLST) data. Infect Genet Evol 2006, 6:97-112.
38. Smith JM, Dowson CG, Spratt BG: Localized sex in bacteria. Nature 199I, 349:29-31.

39. Milkman R, Mckane M: DNA sequence variation and recombination in E. coli. In Population Genetics of Bacteria Edited by: Baumberg S, Young JPW, Wellington EMH, Saunders JR. Cambridge: Cambridge University Press; 1995: 127-142.

40. Milkman R: Recombination and population structure in Escherichia coli. Genetics 1997, 146:745-750

4I. Mayr E: Systematics and the origin of species New York, NY: Columbia University Press; 1942

42. Deng L, Hayes PK: Evidence for cyanophages active against bloom-forming freshwater cyanobacteria. Freshwater Biol 2008, 53:1240-1252.

43. Rhorlack T, Edvardsen B, Skulberg R, Halstvedt CB, Utkilen HC, Ptacnik R, Skulberg OM: Oligopeptide chemotypes of the toxic freshwater cyanobacterium Planktothrix can form sub-populations with dissimilar ecological traits. Limnol Oceanogr 2008, 53:1279-I293.

44. Utkilen $\mathrm{H}$, Gjølme N: Iron-stimulated toxin production in Microcystis aeruginosa. Appl Environ Microbiol 1995, 61:797-800.

45. Babica $P$, Bláha $L$, Maršálek $B$ : Exploring the natural role of microcystins-a review of effects onphotoautotrophic organisms. J Phycol 2006, 42:9-20.

46. Schatz D, Keren Y, Vardi A, Sukenik A, Carmeli S, Börner T, Dittmann $E$, Kaplan A: Towards a clarification of the biological role of microcystins, a family of cyanobacterial toxins. Environ Microbiol 2007, 9:965-970.

47. Otsuka S, Suda S, Li R, Matsumoto S, Watanabe MM: Morphological variability of colonies of Microcystis morphospecies in culture. J Gen Appl Microbiol 2000, 46:39-50.

48. Otsuka S, Suda S, Li R, Watanabe M, Oyaizu H, Hiroki M, Mahakhant A, Liu Y, Matsumoto S, Watanabe MM: Phycoerythrin-containing Microcystis isolated from P.R. China and Thailand. Phycol Res 1998, 46(Suppl 2):45-50.

49. Mes TH, Doeleman M, Lodders N, Nübel U, Stal LJ: Selection on protein-coding genes of natural cyanobacterial populations. Environ Microbiol 2006, 8: I534-I543.

50. Whitaker RJ, Grogan DW, Taylor JW: Geographic barriers isolate endemic populations of hyperthermophilic archaea. Science 2003, 301:976-978.

51. Papke RT, Ward DM: The importance of physical isolation to microbial diversification. FEMS Microbiol Ecol 2004, 48:293-303.

52. Hanage WP, Spratt BG, Turner KM, Fraser C: Modelling bacterial speciation. Philos Trans R Soc Lond B Biol Sci 2006, 361:2039-2044.

53. Fraser C, Hanage WP, Spratt BG: Recombination and the nature of bacterial speciation. Science 2007, 3 I 5:476-480.

54. Falush D, Torpdahl M, Didelot X, Conrad DF, Wilson DJ, Achtman M: Mismatch induced speciation in Salmonella: model and data. Philos Trans R Soc Lond B Biol Sci 2006, 36 I:2045-2053.

55. Baker JA, Entsch B, Neilan BA, McKay DB: Monitoring changing toxigenicity of a cyanobacterial bloom by molecular methods. Appl Environ Microbiol 2002, 68:6070-6076.

56. Thompson JR, Pacocha S, Pharino C, Klepac-Ceraj V, Hunt DE, Benoit J, Sarma-Rupavtarm R, Distel DL, Polz MF: Genotypic diversity within a natural coastal bacterioplankton population. Science 2005, 307:|3||-|3|3.

57. Roumagnac P, Weill FX, Dolecek C, Baker S, Brisse S, Chinh NT, Le TA, Acosta C], Farrar J, Dougan G, Achtman M: Evolutionary history of Salmonella typhi. Science 2006, 3 | 4: |30|-| 304. 